\title{
Low-Cost Joystick for Pediatric Respiratory Exercises
}

\author{
André Sanches Fonseca Sobrinho ${ }^{1}$ (D) Paulo Rogério Scalassara ${ }^{1}$ (D) - María Eugenia Dajer ${ }^{1}$ (i)
}

Received: 1 July 2020 / Accepted: 28 August 2020 /Published online: 14 September 2020

(C) Springer Science+Business Media, LLC, part of Springer Nature 2020

\begin{abstract}
The use of body signals for health care applications has become ubiquitous in the last decade. One utilization of such measurements is the monitoring of respiratory flow for physiotherapy assistance. This application is based on relative flow measures which can rely on inexpensive sensors. Based on that, we present a low-cost electronic device that detects blows and suctions with a pressure sensor and emulates a keyboard for interfacing with computers. This joystick allows children to control free internet games by associating blows and suctions with different intensities to keyboard actions. Also, the intensity can be calibrated according to the user's pulmonary capacities. This feature is adequate for gradual respiratory physiotherapy and can be customized for each patient. In order to verify the operation of the proposed device, practical tests were performed with three online free games, where the joystick functionality was assessed with different therapeutic configurations.
\end{abstract}

Keywords Respiratory exercises $\cdot$ Pediatric patients $\cdot$ Embedded systems $\cdot$ Pressure sensor

\section{Introduction}

The acquisition of body signals for personalized health care assistance is a trend nowadays [50], especially using mobile technologies [21]. The use of wearables and the internet of things is widespread in researches and system design, the focus is on more efficient and accessible care even in remote locations [27]. This is specially true in the case of pandemics, such as COVID-19 [7]. Amongst all the applications of these signals, the acquisition of respiratory flow is less used in monitoring devices because the most common sensors are still too invasive [26].

Due to that, several researches aim at presenting new methods and alternative sensors for respiratory applications

This article belongs to the Topical Collection: Patient Facing Systems

Paulo Rogério Scalassara

prscalassara@utfpr.edu.br

André Sanches Fonseca Sobrinho

andresobrinho@utfpr.edu.br

María Eugenia Dajer

medajer@utfpr.edu.br

1 Department of Electrical Engineering, Federal University of Technology - Paraná, Cornélio Procópio, Brazil in order to assess the health conditions of patients. Examples include a device composed of a pressure sensor array for obtaining respiratory information of people in bed [41] and an alternative respiratory monitoring device that is based on video processing [26]. Such systems are not movable since they need large devices for acquisition and processing of the collected signals. Another example is a more portable system equipped with pressure and pyroelectric sensors designed to monitor respiratory conditions, such as flow and $\mathrm{CO}_{2}$ concentration [48].

However, some applications do not require the exact estimation of the respiratory flow, but merely relative measurements of people's capacity to inspire and expire air. One such application is respiratory physiotherapy, that is a dynamic process with therapeutic application of mechanical interventions based on the physiology of the airways. The main objective of this therapy is to prevent or reduce the consequences of obstruction by secretion in the superior and inferior airways and to increase respiratory work $[6,31]$.

Techniques applied in respiratory physiotherapy commonly make use of mechanical devices that encourage inspiration, such as incentive spirometer, and expiration, such as positive expiratory pressure (PEP) exerciser [20, 34]. The advantages and difficulties of such practices have been widely studied in recent years [33]. This kind of therapy is also commonly used for prevention as is the case of pulmonary complications after abdominal surgeries[35]. 
Patients with respiratory impairment suffer reduction in muscle strength and endurance, respiratory muscle fatigue, and energy consumption. Several studies, such as $[3,8$, 9], have demonstrated that exercise programs and motor and respiratory therapy improve aerobic capacity, breathing pattern, muscle strength, and quality of life in patients with respiratory, swallowing, and voice problems. The use of portable spirometers for e-health, that is, therapy at home with internet assistance, is the focus of studies for some decades [5, 14].

For the specific case of speech therapy, patients perform different blowing and suction exercises in order to treat atypical swallowing, cleft lip and palate, facial paralysis, cerebral paralysis, dysphonia, and in laryngectomized rehabilitation [19, 25, 30]. For those exercises, simple devices composed of bottles and blowing hoses can be used, their goal is to transfer water from one bottle to the other using blows and suctions.

Although these devices and others that measure expiratory flow, like Microlife's PF100 asthma monitor [29], are inexpensive and functional with adults, they have low playful appeal with children, who are not encouraged to use them. That is common even with adults, that must perform repetitive procedures for long periods of time. Studies have shown that the games can be used to assist treatment of chronic diseases [17, 40], especially for children. Because of this, new devices are being designed to entertain and assist the patients during the physiotherapy sessions. Some researchers present examples where spirometers are used in conjunction with games for this purpose $[32,46]$. Also, recent prototypes are in development based on that principle such as Aluna [1] and Playphysio [36], which focus on asthma and cystic fibrosis respectively, and ZephyRx [49] that is a spirometer for breathing exercises.

A recent work [2] presents a study about recreational activities for treatment of pediatric asthma pointing at necessary game features. Their conclusion is that a spirometer based game could assist in that process, while containing costs, especially if it helped the patients realize their treatment progresses.

Also, for cystic fibrosis treatment, the use of games resulted in patient attitude changes [11]. Due to that approach, patients tended to have a better engagement in the therapy. Cystic fibrosis is a disease that causes production of unusual secretions in the lungs resulting in breathing difficulties. Therapy is essential to prolong life and increase its quality.

Since this is a recent approach to such problems, only a few devices using this gamification approach are available. Also, most of them use proprietary games, what limits the possibilities of choice and cause a fast loss of interest for children. Because of that, in this paper, we propose a low-cost electronic device (joystick) that detects blows and suctions and emulates a keyboard for interfacing with computers. With this device, children (and also adults) will be able to control free games available in the Internet, in which the actions of typing on a keyboard can be associated with different intensities of blows and suctions.

Thus, in addition to presenting an interactive and attractive interface for children, the operation of the device can be customized for each patient, according to the recommendation of the physiotherapist or speech therapist and the person's breathing capabilities.

This paper is organized as follows. In "Hardware design" and "Firmware design", the hardware and firmware design for the proposed joystick are described. In "Results and discussion", the experimental results obtained using this device with free online games are presented and discussed. Finally, in "Conclusion", the main conclusions are outlined.

\section{Hardware design}

The embedded system of the proposed joystick for respiratory physiotherapy is summarized by the block diagram of Fig. 1. The pressure sensor is the Freescale MPX5700DP [12], chosen due to its low cost and pressure sensitivity.

The MPX series sensors are widely used in different applications such as examination of the urinary tract [42] and muscle actuators [43]. In the case of study [47], an MPX sensor is employed in the construction of an electronic nose aiming to detect elevated pressure in case of clogs in the system.

The MPX5700DP sensor converts positive (blow) and negative (suction) pressures into positive and negative voltage variations, respectively [16]. By laboratory tests, it was verified that the typical voltage variation at the output of this sensor is between 70 and $300 \mathrm{mV}$, which is the result

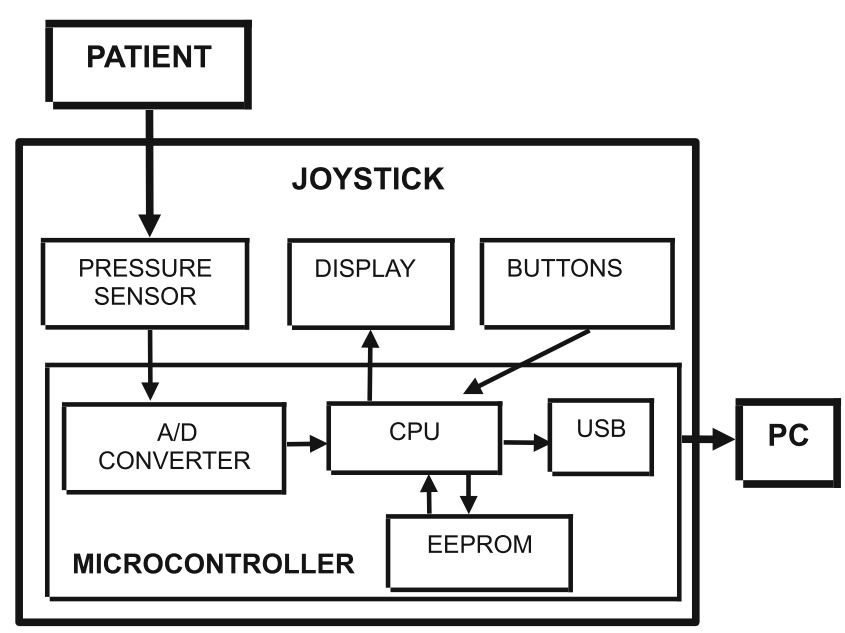

Fig. 1 Block diagram of the joystick embedded system 
of suctions and blows with different intensities in the nozzle. In the absence of these respiratory movements, the voltage varies between 170 and $200 \mathrm{mV}$, which agrees with the expected response of the sensor [12], as illustrated in Fig. 2.

Also, aiming at low cost and the required system functionalities, we chose the Microchip PIC18F4550 microcontroller [28]. It performs the analog-to-digital conversion of the pressure sensor output using its internal 10bit analog-to-digital (A/D) converter. It was not necessary to amplify the sensor signal before acquisition because the A/D converter resolution combined with the linear response of the pressure sensor were sufficient to accurately distinguish the different intensity levels of blows and suctions.

According to the converted level, the microcontroller sends different characters to the PC, emulating a keyboard with its USB interface [18]. The relationship between the converted level and the desired keyboard key is configured using four buttons, labeled BUTTON1 to BUTTON4, and information shown on an alphanumeric display connected to the microcontroller, according to the diagram of Fig. 1. Details of the firmware are shown in "Firmware design".

The voltage supply of the microcontroller, display, and pressure sensor is provided by the USB interface with the PC. It is sufficient for the complete operation of the device [22], thus the use of an external power supply is not required.

The combination of sensors with the PIC18 family microcontroller is shown to be a functional and lowcost solution in other works. Examples are monitoring of animal health [23], design of a walking stick assistant for people with visual deficiencies [39], and project of assistive handlebar using tactile sensors [44]. Also, in [15], this microcontroller was employed in the design of a mechanical ventilator synchronized with a pulse oximeter.

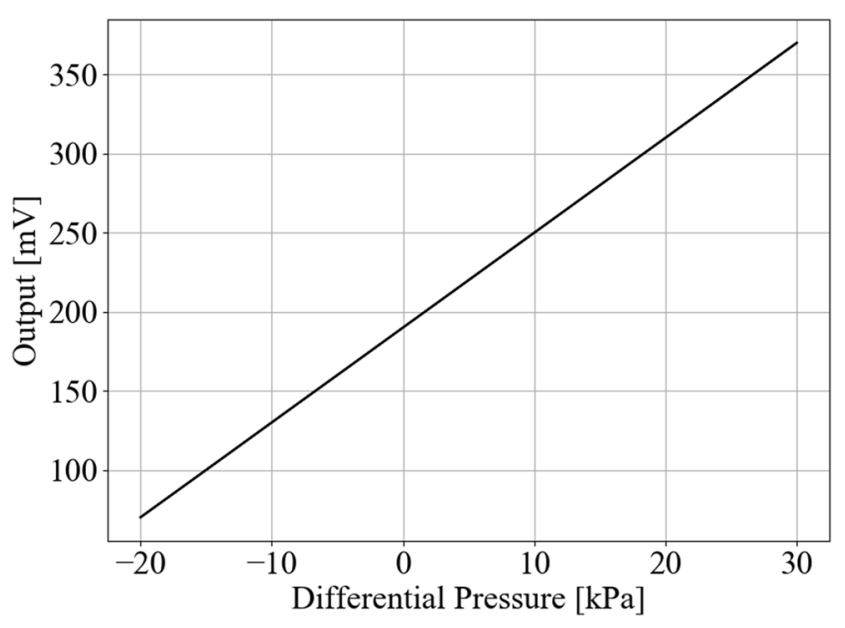

Fig. 2 Expected pressure sensor response [12]

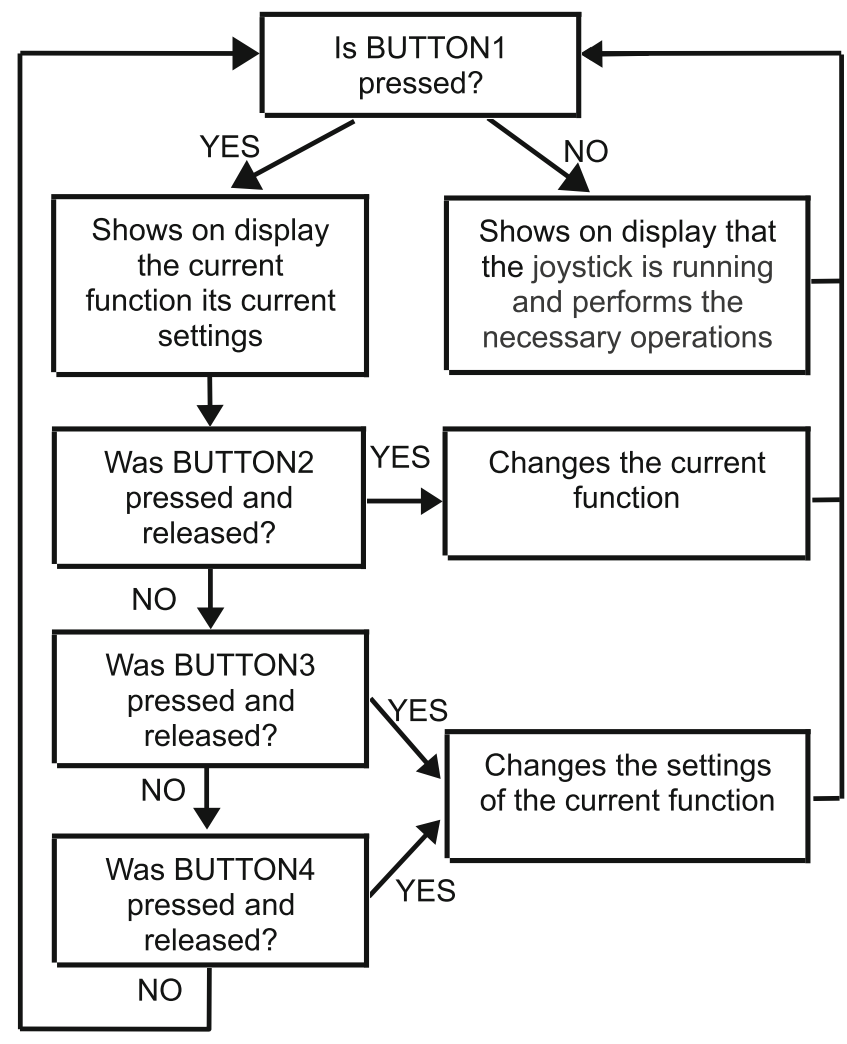

Fig. 3 Block diagram of the firmware design

\section{Firmware design}

The firmware embedded in the microcontroller can be described by the block diagram of Fig. 3. More details of the firmware design are presented in the next sections.

\section{Settings mode}

When BUTTON1 is pressed, the firmware executes the Settings Mode and the message of Fig. 4 appears on the display. The first available function, Blow Key, is presented to the user with its default setting of 1 . This means that, when a blow occurs, the 1 key will be emulated via USB for the $\mathrm{PC}$.

If BUTTON3 is pressed, this value is incremented, for the example of Fig. 4, the value would change to 2 . If

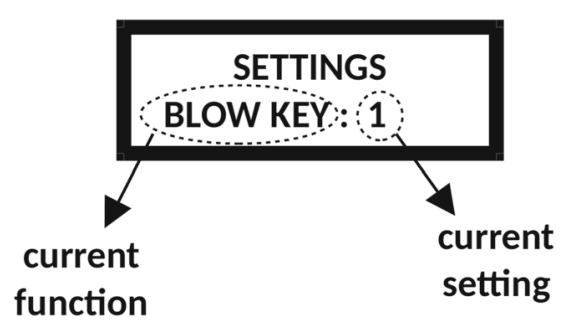

Fig. 4 Settings Mode message 


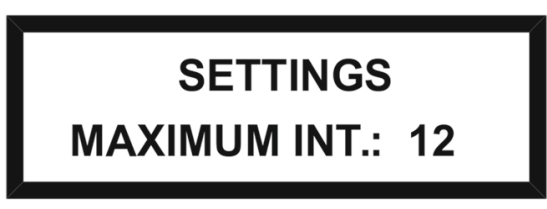

Fig. 5 Blow Calibration message

BUTTON4 is pressed, the value is decremented; again, for the example, the value would change to 0 . The possible values for the Blow Key function are keys 1-9, A-Z, UP, DOWN, RIGHT, LEFT, SPACE, BACK, ENTER, PGDN, and PGUP. If BUTTON2 is pressed, the current function is changed. In addition to the Blow Key function, there are other seven functions available:

1. Suction Key: when suction occurs, the key configured in this setting is emulated via USB for the PC. The possible values are the same as for theBlow Key function.

2. Blow Calibration: in this function, it is not possible to perform settings using BUTTON3 and BUTTON4. The user blows as strong as possible and the maximum intensity of his blow is acquired and presented on the display (Fig. 5). Later, in this section, we present more details about this function.

3. Suction Calibration: similar to the Blow Calibration function, the user suctions as strong as possible and the maximum intensity of his suction is detected and presented on the display. Again, more details are presented later in this section.

4. Blow Intensity: the user sets the blow intensity necessary to activate the key action, Fig. 6. This key was previously selected in the Blow Key function. The possible settings for this function, using BUTTON3 and BUTTON4, are values ranging from 1 to the maximum intensity previously obtained by the Blow Calibration function.

5. Suction Intensity: similarly to the Blow Intensity function, the user sets the suction intensity needed to emulate the key selected previously by theSuction Key

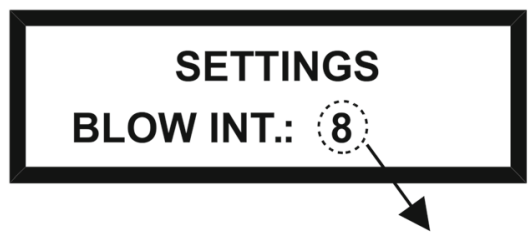

blow intensity

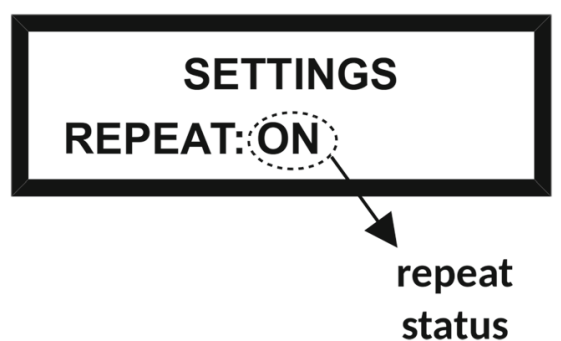

Fig. 7 Repeat message

function. The possible settings for this function are values ranging from 1 to the maximum intensity obtained previously by the Suction Calibration function.

6. Repeat Mode: in this function, it is possible to enable or disable, using BUTTON3 and BUTTON4, the continuous transmission of a key for the PC (Fig. 7) during a blow or suction. More details about this function are presented later in this section.

7. Time Duration: in this function, it is possible to choose time duration $(0,1,2,3$, or 4 seconds), using BUTTON3 and BUTTON4, required to validate the occurrence of a blow or suction (Fig. 8). More details about this function are shown later in this section.

These settings are stored in the microcontroller internal non-volatile memory. Therefore, when the system is turned on, the previously chosen setting values are recovered.

If the Blow and Suction Calibrations functions are selected, the microcontroller, using its A/D converter, waits for a variation of the pressure sensor output voltage. As the 10-bit converter operates in a range between 0 and $5 \mathrm{~V}$, its voltage resolution is $4.883 \mathrm{mV}$.

It is important to note that the A/D converter operating in this voltage range already guarantees sufficient resolution. Therefore, it is not necessary to configure the microcontroller analogically with another operating range, thus reducing the size and cost of the hardware design.

According to the sensor characteristics [12], its output achieves a maximum of approximately $200 \mathrm{mV}$ on standby. When the voltage is greater than this value, the start of a blow is identified. In this situation, if the Blow Calibration function is selected, the microcontroller reads the analog

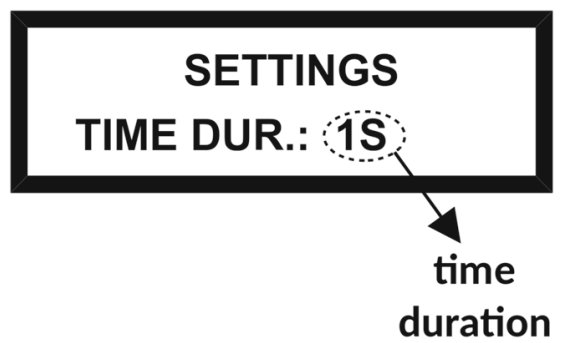

Fig. 8 Time Duration message

Fig. 6 Blow Intensity message 


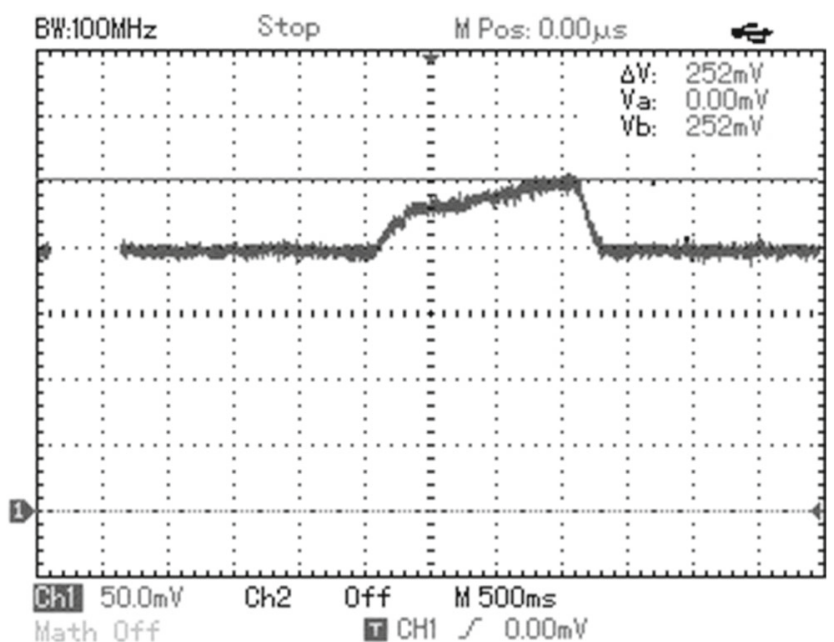

Fig. 9 Maximum voltage value (maximum blow intensity) during the Blow Calibration function

input during 3 seconds. Then the highest level reached during the blowing is obtained, which corresponds to the maximum blow intensity mentioned before.

For the sensor maximum value on standby, the microcontroller converts the $200 \mathrm{mV}$ to level 41, according to Eq. (1).

Level $=\left\lfloor\frac{200 \mathrm{mV}}{\text { Resolution }}\right\rceil=\left\lfloor\frac{200 \mathrm{mV}}{4.883 \mathrm{mV}}\right\rceil=41$

As an example of the sensor output voltage variation during a Blow Calibration, if the maximum voltage detected is $252 \mathrm{mV}$, the $\mathrm{A} / \mathrm{D}$ converter obtains level 52 . This is illustrated in Fig. 9, which shows an oscilloscope screen capture of the sensor voltage signal. Based on that, the blow intensity level is the difference between the obtained maximum level of 52 and the standby reference of 41 , resulting in 11 .

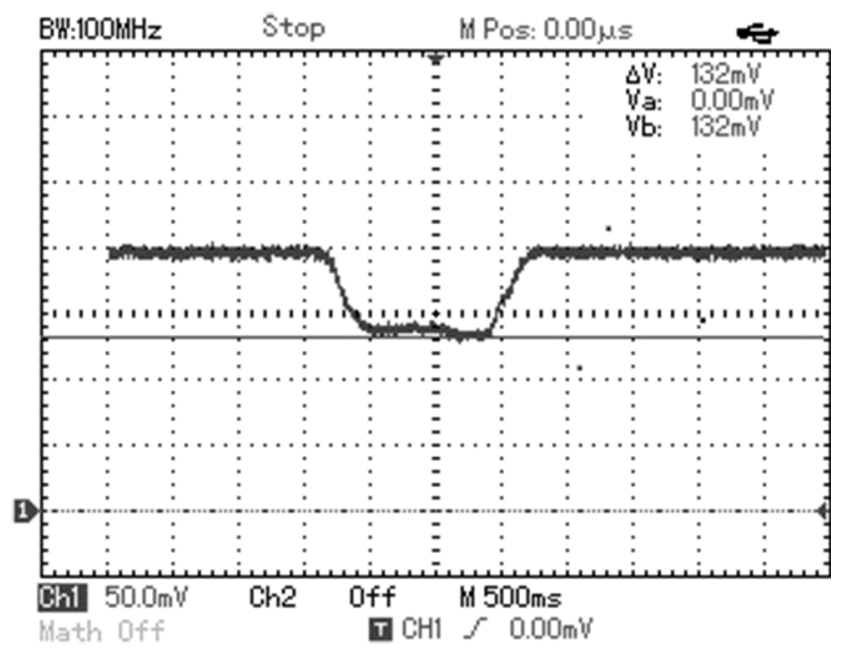

Fig. 10 Minimum voltage value (maximum suction intensity) during the Suction Calibration function

\section{IN OPERATION}

Fig. 11 Operation Mode message

Similarly, when the sensor output voltage is lower than $170 \mathrm{mV}$ (level 35), the minimum voltage on standby, the start of a suction is identified. If the Suction Calibration function is selected, the microcontroller reads the analog input during 3 seconds, obtaining the lowest pressure level during suction, which corresponds to the maximum suction intensity mentioned before.

Figure 10 shows an oscilloscope screen capture of the sensor output signal for a suction example that reached a minimum voltage of $132 \mathrm{mV}$, which corresponds to level 27. The suction intensity level is calculated as the difference between the minimum standby level of 35 and the obtained minimum suction value of 27 , thus the maximum suction intensity is 8 .

\section{Operation mode}

When BUTTON1 is released, the system executes the Operation Mode and the message of Fig. 11 is presented on the display. Similarly to what was previously described for the Blow and Suction Calibration functions, the microcontroller waits for a variation of the pressure sensor output voltage.

If the sensor voltage is equal to or greater than the corresponding level previously set by the Blow Intensity function, the system emulates the behavior of a keyboard and transmits, via USB interface, the key set in the Blow

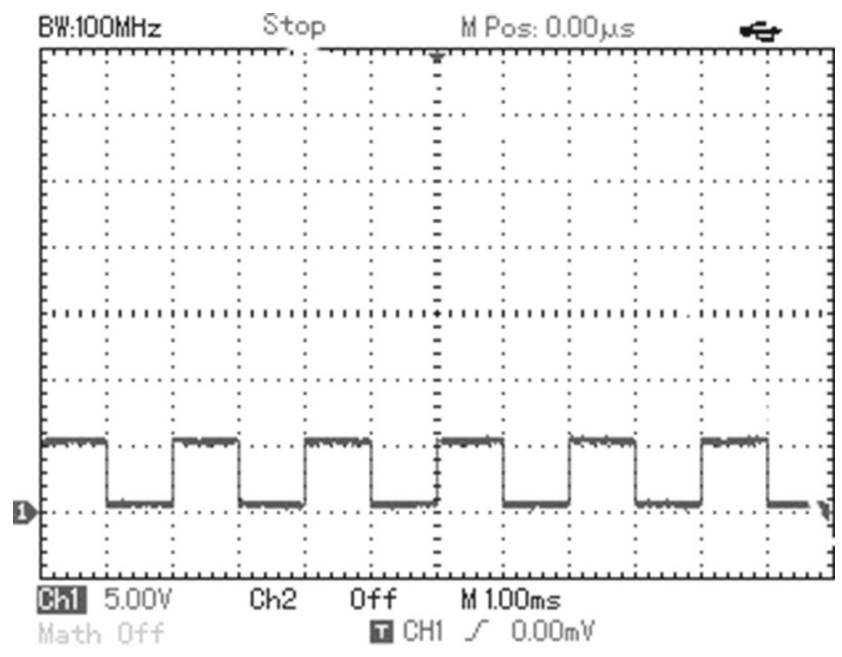

Fig. 12 Time base for the level acquisition and key transmission to the PC 


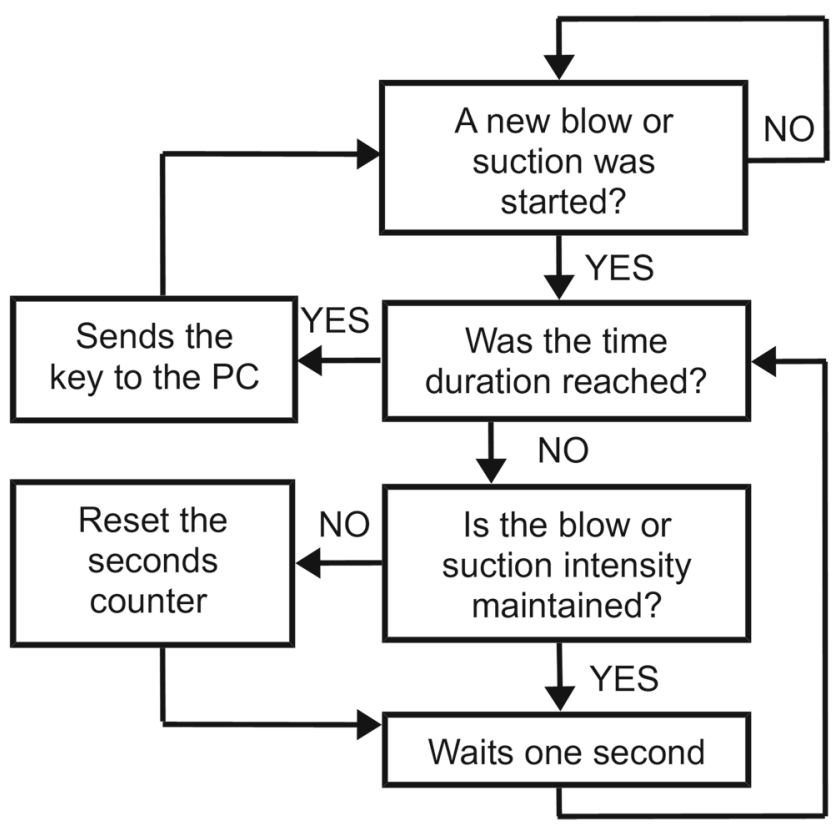

Fig. 13 Block diagram for key transmission

Key function to the computer. Likewise, if the sensor output is equal to or less than the corresponding level previously set by the Suction Intensity function, the microcontroller transmits the key set in the Suction Key function to the computer. The process that comprise the acquisition and transmission of a key to the computer via the USB interface spend a total time of $1 \mathrm{~ms}$. This is shown by the oscilloscope screen capture of Fig. 12, where the logic level is alternated on the microcontroller pin.

If the Repeat function is on, the key represented by the obtained sensor level is sent to the PC repeatedly every $1 \mathrm{~ms}$ while the intensity of the blow or suction is maintained. In this mode, the configuration of the Time Duration function is not performed. However, if Repeat function is off, only one key is sent to the $\mathrm{PC}$ if the time duration and the intensity of the blow or suction are respected. In this case, even if the time duration of blowing or sucking is

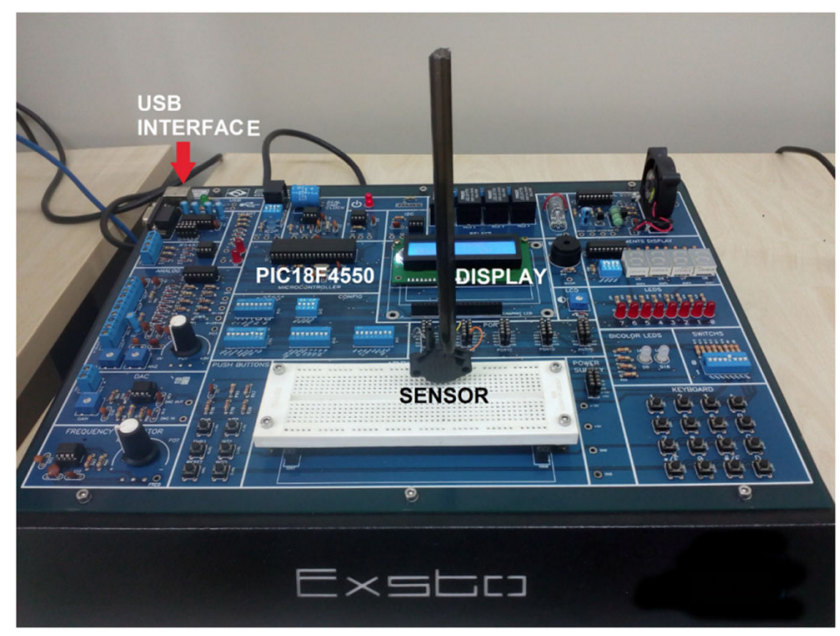

Fig. 15 Exsto XM118 development kit with the MPX5700 pressure sensor

greater than what was set, only one key is transmitted. This procedure is described by the block diagram of Fig. 13.

The seconds counter shown in Fig. 13 is based on a 16bit timer of the microcontroller, which allows up to 65536 counts. The cycle tick, based on a $20 \mathrm{MHz}$ crystal, was configured to $25.6 \mu \mathrm{s}$. Therefore, when the timer reached count 39062, a time period equal to $0.9999987 \mathrm{~s}$ has elapsed, which presented sufficient accuracy to guarantee that the time duration of a blowing or a suction had been adequately acquired.

\section{Results and discussion}

The functional results of the low-cost joystick for respiratory exercises were obtained by practical tests using the setup presented in Fig. 14, which is composed of a personal computer, MPX5700 pressure sensor, and the microcontroller development kit, model XM118, manufactured by Exsto [10].

This development kit (Fig. 15) was chosen to validate the proposed system because it has, in the same platform,

Fig. 14 Setup for practical tests

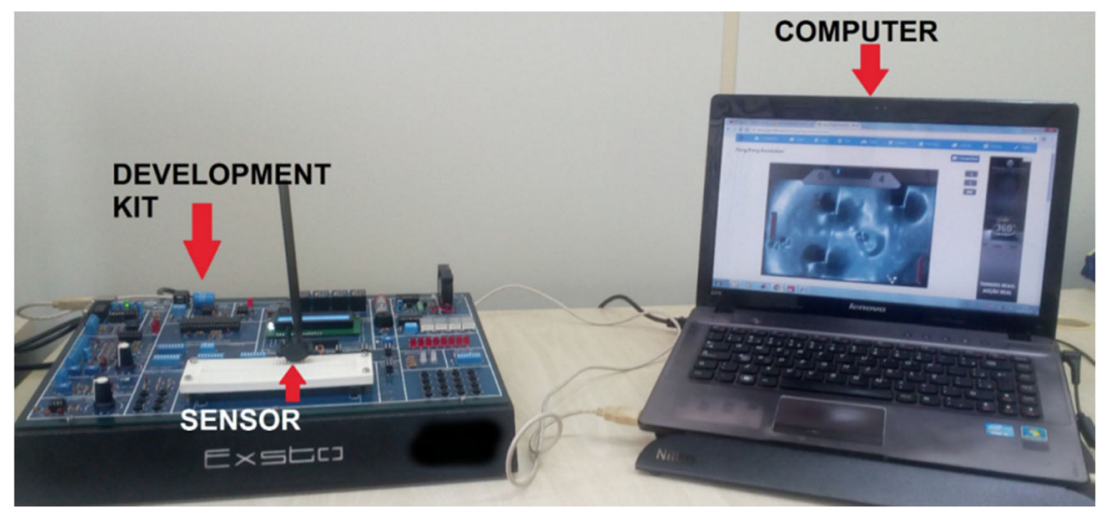




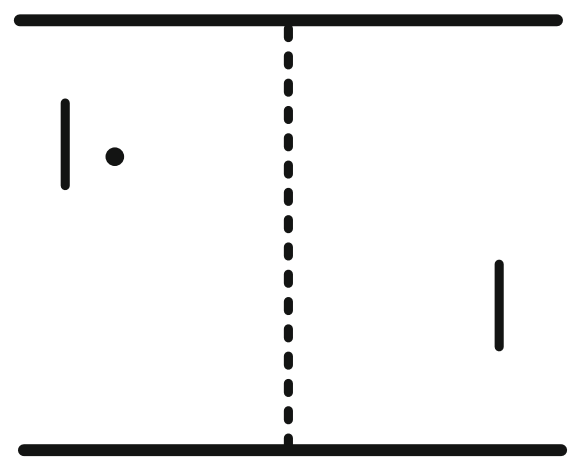

Fig. 16 Pong gameplay example screen

the microcontroller, its programmer, display, buttons, USB interface, and prototype board, in which the pressure sensor was interfaced.

In the next sections, we present initial tests using the proposed joystick with three free games available online: Pong, Tetris, and Minigolf. In each game, a specific configuration of repetition and duration of blow/suction is used to demonstrate the possibilities of respiratory physiotherapy, in which the verification of compliance with the parameters previously configured during the game was possible using an oscilloscope.

In addition, the complexity of the games increases with each test, in order to demonstrate that the proposed device can be used with games with different playing mechanics. This characteristic is important for a joystick aimed at children, that can be easily bored by simple games or lack of options. By focusing on more complex games, some controls had to be ignored or had to be performed at the PC keyboard. Despite that, the device still allows the execution of physiotherapy with such games.

\section{First test using a Pong game}

In a Pong game, the player must score points using his virtual racket that slides up and down as illustrated in Fig. 16. Games like that can be played for free on several websites. Usually, it is possible to configure the keyboard keys that slide the racket up and down, which can be moved continuously by holding down the configured key.

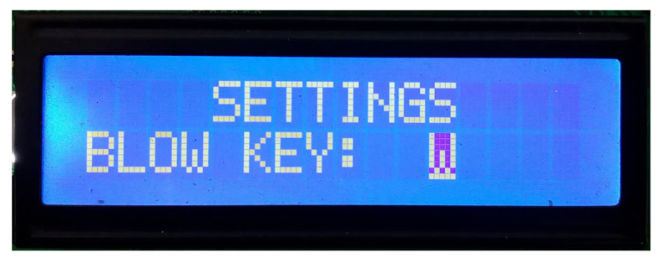

Fig. 17 Display with the Blow Key function being configured as the W key

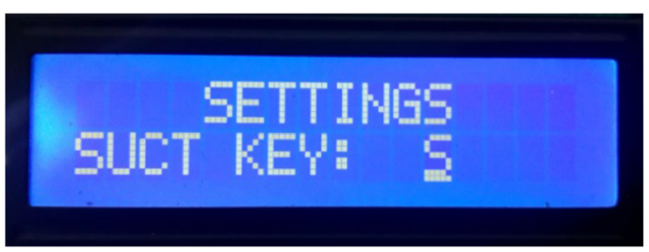

Fig. 18 Display with the Suction Key function being configured as the S key

This kind of game is interesting for respiratory physiotherapy because the patient has to perform continuous blows or suctions in order to move the racket appropriately. In some cases where the physiotherapy is recommended for prevention of respiratory complications, like after abdominal surgery [38], intermittent breathing is not effective. Thus, the configuration presented in this test can assist the treatment. Also, it can be easily personalized to the patient's pulmonary capacities using the Blow/Suction Calibration functions. This procedure is described in the following paragraphs.

In order to play the game with the proposed joystick, the first step was pressing BUTTON1 for the system to enter the Settings Mode. Afterwards, as an example, the W key was set in the Blow Key function, as shown in Fig. 17, using BUTTON3 and BUTTON4.

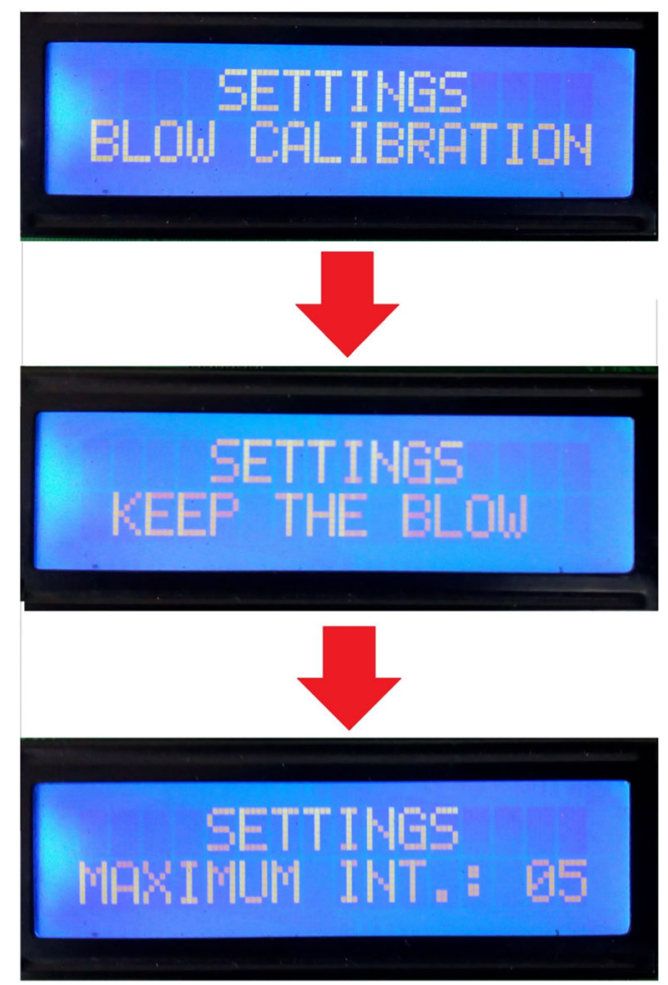

Fig. 19 Display with the Blow Calibration messages 

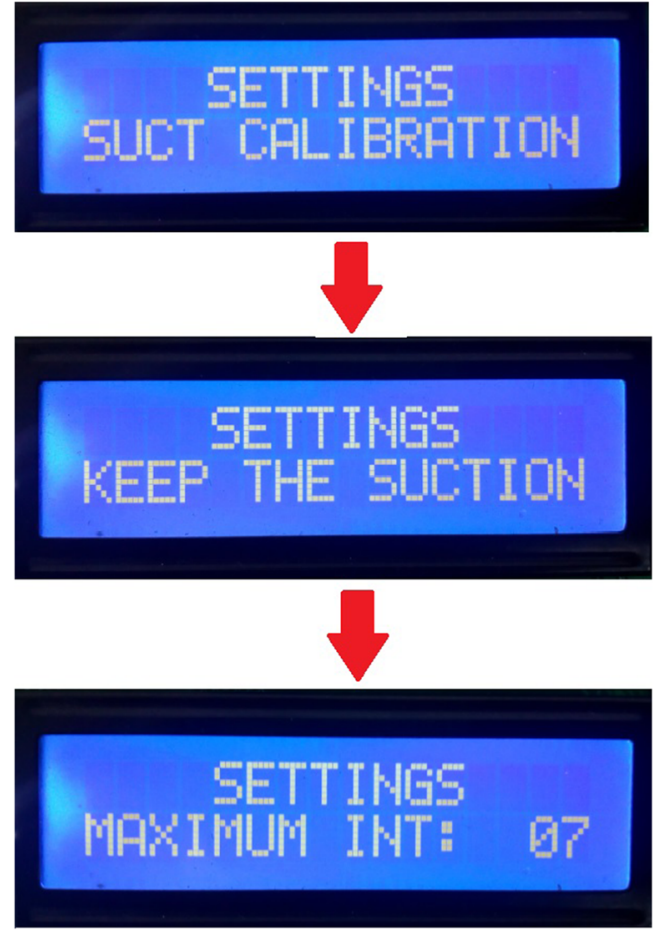

Fig. 20 Display with the Suction Calibration messages

Next, the Suction Key function was selected, using BUTTON2, and configured as the $\mathrm{S}$ key. This example is illustrated in Fig. 18.

Then, the Blow Calibration function was selected. Figure 19 shows the sequence of messages on the display during the execution of this function, where the maximum Blow Intensity obtained in this example was 5.

Similarly, when the Suction Calibration function was executed, the sequence of messages presented by Fig. 20

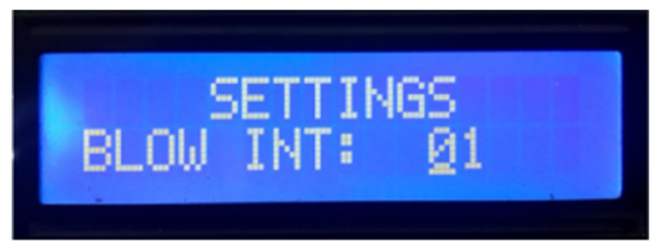

(a)

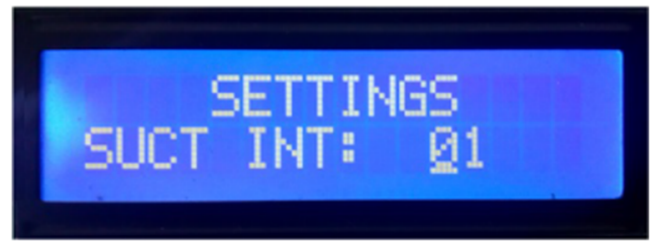

(b)

Fig. 21 Display with the (a) Blow Intensity and (b) Suction Intensity functions configured to minimum

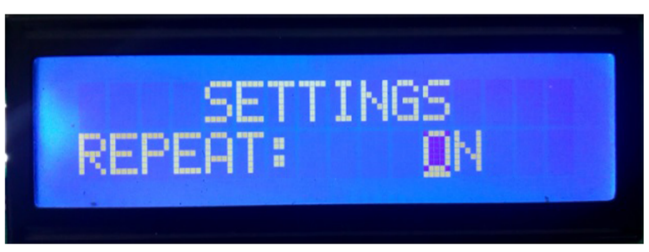

Fig. 22 Display with the Repeat function set as on

was displayed, with an example of 7 for the maximum Suction Intensity.

Later, as shown in Fig. 21 (a) and (b) respectively, the lowest possible intensities for the execution of blows and suctions were set by the Blow and Suction Intensity functions. These options were configured to a minimum because, in this game, it is necessary to blow and suck continuously to slide the racket, generating high effort.

The Repeat function was set as on, Fig. 22, again because of the necessity to blow and suck continuously, as previously explained. This configuration results in a more dynamic response of the racket slide using the joystick. By releasing BUTTON1, the Operating Mode was executed, displaying the message of Fig. 23.

During this test, the expected operation of the joystick was verified with the aid of an oscilloscope. When the voltage generated by the pressure sensor was greater than $204.83 \mathrm{mV}$ during a blow, corresponding to the minimum configuration level of 1 , the racket slid up continuously. Similarly, when the voltage was less than $165.17 \mathrm{mV}$ during a suction, also for the minimum level of 1 , the racket slid down continuously.

\section{Second test using a Tetris game}

The goal of the Tetris game is to score as many points as possible by clearing horizontal lines of blocks as illustrated in Fig. 24 for the example of the Tetris n-blox game, which can be played for free at the website www.freetetris.org. The player must rotate, move, and drop the falling Tetriminos inside the matrix (playing field). Lines are cleared when they are filled with blocks and have no empty spaces.

Differently from the previous test, in this one, the patient is supposed to take short and intermittent blows/suctions to achieve success in the game. This kind of exercise is recommended, for instance, for patients with COPD

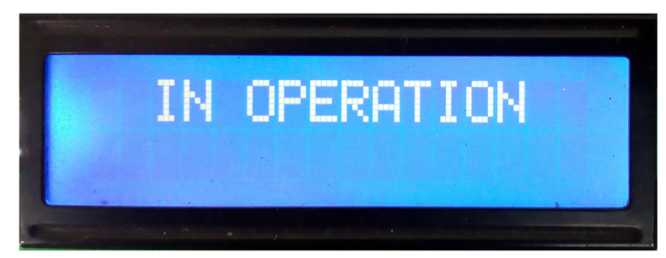

Fig. 23 Display with the Operation Mode message 


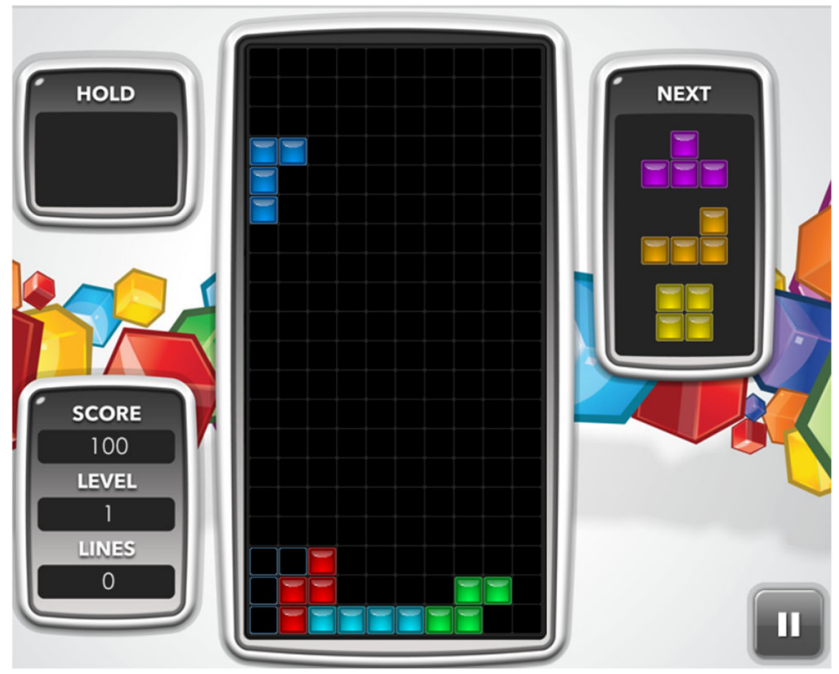

Fig. 24 Tetris n-blox gameplay example screen. Reproduced with permission of the owner

(chronic obstructive pulmonary disease), a common lung disease that makes it harder to breathe, with acute exacerbation, where there are secretions and the person cannot perform an effective cough [4]. Again, as in the previous test, it is possible to calibrate the device for the patient's capabilities.

Figure 25 shows the control setup screen with examples of keys that can be set to control the Tetriminos. The controls were configured as: right arrow (move right), left arrow (move left), up arrow (rotate right), $\mathrm{Z}$ (rotate left), down arrow (soft drop), and space (hard drop). Similarly to

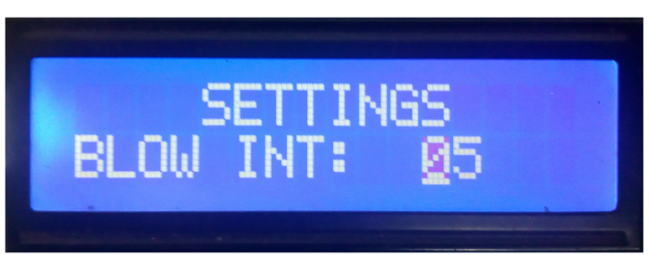

Fig. 26 Display with the Blow Intensity function set to the maximum intensity

the previous example, other keys can be set to perform these controls.

In order to play the game with the proposed joystick, the rotate left and soft drop controls were configured as blow and suction respectively. The move right and move left commands are still controlled by the computer keyboard. The rotate right and hard drop commands were ignored because they are not essential to the game operation.

As shown in the previous example, the first step was executing the Settings Mode. Then, the up arrow key was set in the Blow Key function and the down arrow was set in the Suction Key function. The Blow and Suction Intensity functions were configured to 5 and 7 respectively, as shown in Figs. 26 and 27, according to the maximum intensities obtained previously by the calibration functions (Figs. 19 and 20).

As mentioned before, the purpose of this test is to force the user to perform short blows and suctions with the greatest possible intensities in order to rotate and drop the Tetraminos. Therefore, for each command to fall or rotate, it is necessary to perform one blow or suction. Thus, the
Fig. 25 Tetris n-blox control setup screen. Reproduced with permission of the owner

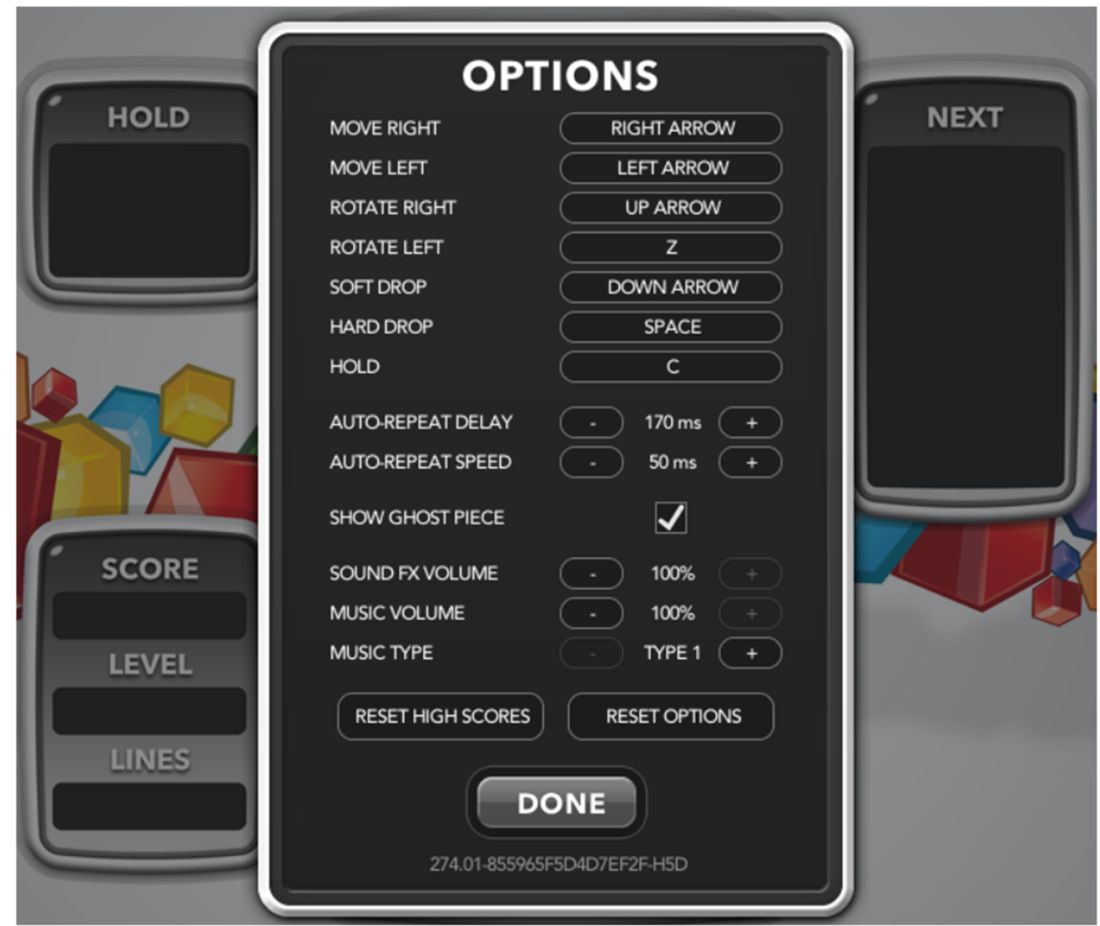




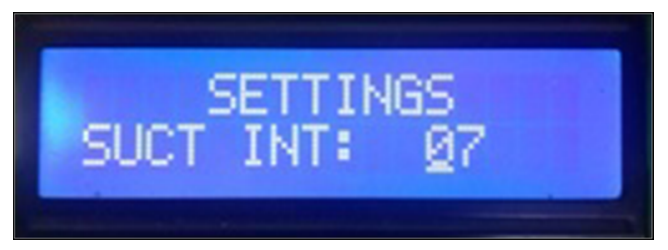

Fig. 27 Display with the Suction Intensity function set to the maximum intensity

Repeat function was set to off to ensure that only one key ( $\mathrm{Z}$ or down arrow in the example) was transmitted to the PC for each action. In order for this procedure to occur as soon as possible, the Time Duration function (Fig. 28) was set to 0 seconds.

As was performed for the Pong game, during the testing, the expected joystick operation was verified using an oscilloscope. However, in this case, the blow and suction intensities where configured to 5 and 7 , respectively. Thus, when the voltage generated by the pressure sensor was greater than $224.42 \mathrm{mV}$ during a blow, corresponding to the maximum level of 5 , the Tetriminos was rotated left. Also, when the voltage was less than $145.58 \mathrm{mV}$ during the suction, equivalent to the maximum level of 7 , the Tetriminos dropped. These actions occurred independently from the duration of the blow or suction.

\section{Third test using a Minigolf game}

In a minigolf game, the player must hit the hole in a minigolf course with as few shots as possible. Games like that can be played for free on several websites. Usually, the keys to control the game are: turn right, turn left, increase shot power, decrease shot power, and perform a shot. In this game, the proposed joystick was used to perform the shot command by a blow and the other commands are still controlled by the keyboard. Again, the first step was executing the Settings Mode, then the space key was set in the Blow Key function.

The purpose of this test is to force the patient to perform long blows with the greatest possible intensity in order to perform a shot. This kind of exercise, PEP (positive expiratory pressure), is indicated to increase lung volume, measured by the functional residual capacity [33]. Thus, the maximum intensity setting of the joystick was maintained in

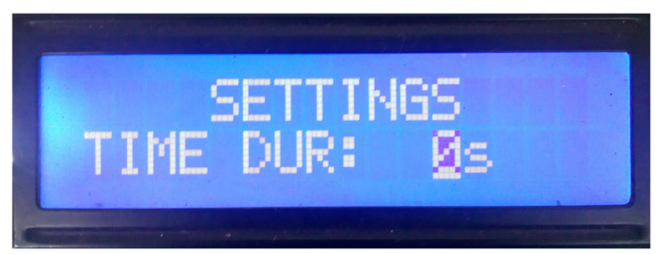

Fig. 28 Display with the Time Duration function set to 0 seconds the Blow Intensity function and the Time Duration function was set to 4 seconds.

Similarly to the other tests, the expected joystick operation was verified with an oscilloscope. During a blow, when the voltage generated by the pressure sensor was greater than $224.42 \mathrm{mV}$, corresponding to level 5 (the maximum achieved by the user in these tests), with an uninterrupted duration of $4 \mathrm{~s}$, a shot was performed.

After the presented tests using three games with different mechanics, the proposed joystick performed as expected, there were no significant detection errors of the user's executed actions. In these initial tests, the only encountered issue was that there is a necessary training period, after which the user can achieve a satisfactory gaming experience, mainly in relation to blowing and sucking through the sensor nozzle. However, for the user, this issue can be interpreted as the usual challenge related to mastering a new game, since it is overcome with some practice.

\section{Conclusion}

The tests performed with the proposed device show the expected therapeutic behavior when the joystick is used for different situations and with different free games. For instance, in procedures aiming to force the patient to perform continuous blow and suction with low intensity, to perform repeatedly short blows and suctions with great intensity, or to perform long blows with great intensity and long pauses.

Joysticks with USB interface in which keyboard actions can be emulated by blowing and sucking, are already available on the market [37]. This kind of device can be found in different patent bases, such as LATIPAT [24] and the United States Patent and Trademark Office [45]. An example is patent number US9436277B2 that presents a system to produce control signals from breath attributes [13].

The drawback is that they do not allow the association of different levels of intensity and periods of blowing and sucking with the actions of the keyboard, and cannot be properly used as a therapeutic tool. As mentioned in the Introduction, there are also other spirometer projects that connect to games on computers or smartphones [1, 32, 46]; however, there are a limited number of games and they are personalized for the devices, without the possibility to interface with free online games.

Considering these limitations of the available devices in the market, the proposed joystick is an interesting option that is focused on respiratory physiotherapy but with the possibility to interface with several games with no extra charge. Also, the capacity to easily calibrate the 
blow/suction intensity for each patient allows a personalization of their treatment. As described with the three tests of "Results and discussion", the health professional can configure the type of respiratory exercise, continuous or intermittent, allowing the execution of a diverse number of physiotherapy practices. These characteristics are recommended in the literature [4], especially complying with the expected features of spirometer based games [2].

It is important to note that in order to use the proposed device, there is no need to install drivers or extra software on the computer, which makes it easier to use when a final model is available.

Finally, the proposed joystick is still a prototype and only initial tests were performed with healthy people, that did not need respiratory physiotherapy. The gaming experience was satisfactory and no issues were detected with the joystick usage, except the necessary initial training period to adapt to a new way to control the game. The next step of this research is to evaluate the performance of the device with physiotherapy patients, especially children, assessing the treatment effectiveness and the system ability to entertain during the exercises. Another future work is to create a registry with the activities performed by the patients in order to integrate the device with e-health solutions as proposed in the literature [14].

Acknowledgements The authors thank the support of the Federal University of Technology - Paraná

Funding This work was supported in part by the Coordenação de Aperfeiçoamento de Pessoal de Nível Superior - Brasil (CAPES) Finance Code 001

\section{Compliance with Ethical Standards}

Conflict of interests The authors declare they have no conflict of interest

Human Participants and/or Animals This article does not contain any studies with human participants or animals performed by any of the authors

\section{References}

1. Aluna: doctor-approved health platform to track child asthma symptoms, www.aluna.io. (accessed June 26, 2020).

2. Bingham, P.M., Crane, I., Manning, S.W.: Illness experience, self determination, and recreational activities in pediatric asthma. Games Health J 6(2), 1-8 (2017).

3. Bisaccioni, C., Aun, M.V., Cajuela, E., Kalil, J., Agondi, R.C., Giavina-Bianchi, P.: Comorbidities in severe asthma: frequency of rhinitis, nasal polyposis, gastroesophageal reflux disease, vocal cord dysfunction and bronchiec-tasis. Clin 64, 769-773 (2009).

4. Bott, J., Blumenthal, S., Buxton, M., et al.: Guidelines for the physiotherapy management of the adult, medical, spontaneously breathing patient. Thorax 64 (Suppl. I), i1-i51 (2009).
5. Bruderman, I., Abboud, S.: Telespirometry: Novel system for home monitoring of asthmatic patients. Telemed J 3(2), 127-133 (1997).

6. Bruton, A., Garrod, R., Thomas, M.: Respiratory physiotherapy: towards a clearer definition of terminology. Physiother J 97(4), 345-349 (2011).

7. Bryant, M.S., Fedson, S.E., Sharafkhaneh, A.: Using telehealth cardiopulmonary rehabilitation during the covid-19 pandemic. J Med Syst 44, 125 (2020).

8. Cress, M.E., Buchner, D.M., Prohaska, T., Rimmer, J., Brown, M., Macera, C., DePietro, L., Chodzko-Zajko, W.: Physical activity programs and behavior counseling in older adult populations. Med. Sci. Sports Exerc. 36, 1997-2003 (2004).

9. Decramer, M.: Effects of hyperinflation on the respiratory muscles. Eur Respir J 2, 299-302 (1989).

10. Exsto Tecnologia Ltd.: XM118 PIC18F4550 microcontroller test kit (2009). https://exsto.com.br/kitdidatico/eletronica/ xm118-microcontroladores-pic18 (accessed June 26, 2020).

11. Ferguson, B., Williams, A.J., Williams, R.H., Bingham, P., Christenson, K.M.: Games for cystic fibrosis: Creating the will to get better (roundtable discussion). Games Health J 1(2), 90-95 (2012).

12. Freescale Semiconductor Inc., Chandler, AZ, USA: MPX5700 data sheet (2012).

13. Furst, M.R., Bernal, E.A., Loce, R.P., Mestha, L.K.: System and method for producing computer control signals from breath attributes (2014). Patent no. US9436277B2.

14. Giansanti, D., Maccioni, G.: Toward the integration of devices for pulmonary respiratory rehabilitation in telemedicine and e-health. Telemed J E Health 25(3), 257-259 (2019).

15. Gölcük, A., Işik, H., Güler, I.: Design and construction of a microcontroller-based ventilator synchronized with pulse oximeter. J Med Syst 40, 180 (2016).

16. Heeley, D.: Application note AN1573 - Understanding pressure and pressure measurement. Freescale Semiconductor Inc., Chandler, AZ, USA (2005).

17. Holtz, B.E., Murray, K., Park, T.: Serious games for children with chronic diseases: A systematic review. Games Health J 7(5), 1-11 (2018).

18. Hosaka, R.: Development of mobile phone type universal human interface device for medical information system. In: R. Magjarevic, J.H. Nagel (eds.) World Congr Med Phys Biomed Eng 2006, vol. 14, pp. 3774-3777. Springer, Berlin, Germany (2006).

19. Howard, S., Lohmander, A.: Cleft palate speech: assessment and intervention. Wiley-Blackwell, West Sussex, UK (2011).

20. Hristara-Papadopoulou, A., Tsanakas, J., and O. Papadopoulou, G.D.: Current devices of respiratory physiotherapy. Hippokratia 12(4), 211-220 (2008).

21. Karageorgos, G., Andreadis, I., Psychas, K., Mourkousis, G., Kiourti, A., Lazzi, G., Nikita, K.S.: The promise of mobile technologies for the health care system in the developing world: a systematic review. IEEE Rev Biomed Eng 12, 100-122 (2019).

22. Kollman, R., Betten, J.: Powering electronics from the USB port. Texas Instruments Inc., Dallas, TX, USA (2005).

23. Kumar, A., Hancke, G.P.: A zigbee-based animal health monitoring system. IEEE Sens J 15(1), 610-617 (2015).

24. LATIPAT patent search. lp.espacenet.com. (accessed June 26, 2020).

25. Machado, J.R.S., Steidl, E.M.S., Bilheri, D.F.D., Trindade, M., Weis, G.L., Jesus, P.R.O., Pereira, M.B., Mancopes, R.: Effects of muscle respiratory exercise in the biomechanics of swallowing of normal individuals. CEFAC 17(6), 1909-1915 (2015).

26. Massaroni, C., Presti, D.L., Formica, D., Silvestri, S., Schena, E.: Non-contact monitoring of breathing pattern and respiratory rate via RGB signal measurement. Sens 19, 2758 (2019). 
27. Metcalf, D., Milliard, S.T.J., Gomez, M., Schwartz, M.: Wearables and the internet of things for health: wearable, interconnected devices promise more efficient and comprehensive health care. IEEE Pulse 7(5), 35-39 (2016).

28. Microchip Technology Inc., Chandler, AZ, USA: PIC18F2455/2550/4455/4550 data sheet (2009).

29. Microlife: PF 100 asthma monitor. www.microlife.com/ consumer-products/respiratory-care/asthma-monitor/pf- 100 . (accessed June 26, 2020).

30. Nagarajan, R., Savitha, V., Subramaniyan, B.: Communication disorders in individuals with cleft lip and palate: an overview. Indian Journal of Plastic Surgery 42, S137-S143 (2015).

31. Oberwaldner, B.: Physiotherapy for airway clearance in pediatrics. Eur Respir J 15(1), 196-204 (2000).

32. Oikonomou, A., Day, D.: Using serious games to motivate children with cystic fibrosis to engage with mucus clearance physiotherapy. In: Proc. 2012 Int. Conf. Complex Intell. Softw. Intensiv. Syst., Palermo, Italy, pp. 34-39 (2012).

33. Olsén, M.F., Lannefors, L., Westerdahl, E.: Positive expiratory pressure - common clinical applications and physiological effects. Respir Med 109, 297-307 (2015).

34. Opdekamp, C., Sergysels, R.: Respiratory physiotherapy in lung diseases. Rev Med Brux 24(4), A231-235 (2003).

35. Pasquina, P., Tramèr, M.R., Granier, J.M., Walder, B.: Respiratory physiotherapy to prevent pulmonary complications after abdominal surgery: a systematic review. Chest 130, 1887-1899 (2006).

36. Playphysio. play.physio. (accessed June 26, 2020).

37. Quadstick: a game controller for quadriplegics. www.quadstick. com. (accessed June 26, 2020).

38. Rezaiguia, S., Jayr, C.: Prevention of respiratory complications after abdominal surgery. Ann Fr Anesth Reanim 15, 623-646 (1996).

39. Sahoo, N., Lin, H.W., Chang, Y.H.: Design and implementation of a walking stick aid for visually challenged people. Sens 19, 130 (2019).
40. Sánchez, I.T., Salmerón, Y.M., López, L.L., Rubio, A.O., Torres, J.R., Valenza, M.C.: Videogames in the treatment of obstructive respiratory diseases: A systematic review. Games Health J 8(4), 237-249 (2019).

41. Sun, C., Li, W., Chen, W.: A compressed sensing based method for reducing the sampling time of a high resolution pressure sensor array system. Sens 17, 1848 (2017).

42. Suryawanshi, A., Joshi, A.: A method to examine functioning and dysfunctioning of lower urinary tract. In: Proc. 2012 IEEE Int. Conf. Ind. Inf. Syst., Chennai, India (2012).

43. Tang, R.: Design of a parallel shoulder assistive robot with pneumatic muscle actuators. Master's thesis, Univ. Technol., Sydney, Australia (2013).

44. Trujillo-León, A., Bachta, W., Castellanos-Ramos, J., VidalVerdú, F.: Assistive handlebar based on tactile sensors: control inputs and human factors. Sens 18, 2471 (2018).

45. United States Patent and Trademark Office (USPTO). www.uspto. gov. (accessed June 26, 2020).

46. Vilozni, D., Barker, M., Jellouschek, H., Heimann, G., Blau, H.: An interactive computer-animated system (SpiroGame) facilitates spirometry in preschool children. Am J Respir Crit Care Med 164(12), 2200-2205 (2001).

47. Wojnowski, W., Majchrzak, T., Dymerski, T., Gȩbicki, J., Namieśnik, J.: Portable electronic nose based on electrochemical sensors for food quality assessment. Sens. 17, 2715 (2017).

48. Yang, J., Chen, B., Zhou, J., Lv, Z.: A low-power and portable biomedical device for respiratory monitoring with a stable power source. Sens 15, 19618-19632 (2015).

49. Zephyrx for your health - video games for respiratory therapy. zephyrx.com. (accessed June 26, 2020).

50. Zhang, S., Bamakan, S.M.H., Qu, Q., Li, S.: Learning for personalized medicine: a comprehensive review from a deep learning perspective. IEEE Rev Biomed Eng 12, 194-208 (2019).

Publisher's Note Springer Nature remains neutral with regard to jurisdictional claims in published maps and institutional affiliations. 